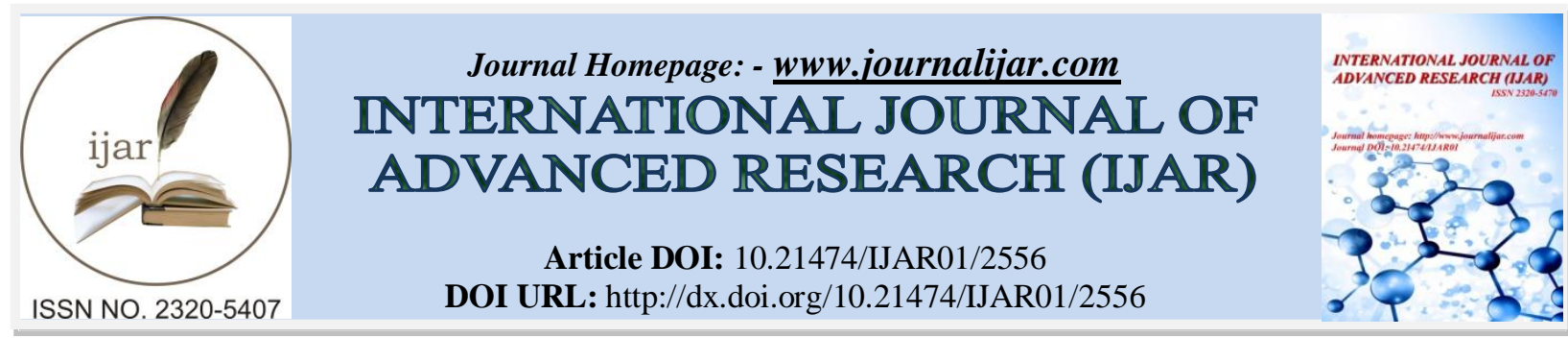

RESEARCH ARTICLE

\title{
EFFECTIVE WIRELESS COMMUNICATION.
}

Ramya. K and Pavithradevi. K.

Assistant Professor, Department of MCA, Gnanamani College of Technology, Namakkal, Tamil Nadu, India.

\section{Manuscript Info}

Manuscript History

Received: 27 October 2016

Final Accepted: 25 November 2016

Published: December 2016

\section{Key words:-}

Wireless Sensor Network, IEEE 802.11 Protocols, Por System, WPAN, Mobile Data Network.

\section{Abstract}

Wireless Sensor Networks (WSNs) have been attracting increasing interest for supporting a new generation of ubiquitous computing systems with great potential for many applications such as surveillance, environmental monitoring, health care monitoring or home automation. However, the communication paradigms in WSNs differ from the ones associated to traditional wireless networks, triggering the need for new communication protocols. In this context, the IEEE 802.11 protocol presents some potentially interesting features for supporting large-scale ubiquitous computing applications, namely pro-efficiency, timeliness and scalability. Nevertheless, when addressing applications with (soft/hard) timing requirements some Inherent paradoxes emerge, such as pro-efficiency versus timeliness. Then, it presents some timing performance analysis that unveils some directions for resolving the previously mentioned paradoxes.

Copy Right, IJAR, 2016,. All rights reserved.

\section{Introduction:-}

Wireless Sensor Networks (WSNs) have revolutionized the design of emerging embedded systems and triggered a new set of potential applications. This particular form of distributed and ubiquitous computing raises many challenges in terms of real-time Communication and coordination to the large number of constraints that must be simultaneously satisfied, including limited pro, CPU speed, storage capacity and bandwidth. These constraints trigger the need for new paradigms in term sensor node /sensor design and network communication/coordination mechanisms.

The design of wireless sensor networks is mainly concerned with pro-efficiency issues, due to the several limitation in terms of energy consumption However, the design complexity is even more significant when applications have, in addition, real-time and/or scalability requirements Several research initiatives, aiming at providing different design solutions for WSNs protocols, have been recently emerged Bandyopadhyay and Hover, believe that the use of standard technologies pushed forward by commercial manufacturers can speed-up a wider utilization of WSNs.

\section{Research trends and challenges of the IEEE 802.11 protocol:-}

Since its proposal in 2003, the IEEE 802.11 protocol has been attracting more and more research work envisaging its deployment in wireless sensor networks. It is expected that many commercial manufacturers of wireless sensor technologies will shift towards this standard solution due to its low-cost and improved performance. Hover, there are still some challenges that must be addressed to tune this COTS technology for WSN applications.

Corresponding Author:- Ramya.K.

Address:- Assistant Professor, Department of MCA, Gnanamani College of Technology, Namakkal,

Tamilnadu, India. 
In this section, present some hints on potential research trends and future challenges for the use of IEEE 802.11 in wireless sensor networks. One of the challenges is the deployment of the beacon-enabled mode in a multi-hop network with several Coordinators. The main problem is to find adequate synchronization schemes for different Coordinators in the same range, to avoid beacon collisions. In such kind of networks, beacon frames of a given Coordinator may collide with beacon frames of other Coordinators or data/control frames. Losing beacon frames will lead to synchronization failures of associated nodes in the network.

This problem was analyzed where the authors derived the probability of beacon frame collisions with other beacons, and the probability of beacon frame collisions with data/control frames. The analytical results and experiments show that, when the starting time of the first beacon frame generated by each Coordinator is uniformly distributed, multihop beacon enabled networks are feasible for $B O$ values higher than 1, and for evenly distributed Coordinators.

\section{Wireless personal area network:-}

WPANs use RF technologies similar to those of WLANs, but are meant for smaller communication coverage areas (10s of meters versus 100s). In 1998, the WPAN group published the original functionality requirement. Also in 1998, the same group invited participation from several organizations, such as Bluetooth source coding is needed at the presentation layer. Finally, at the application layer, one has to conscious about the location dependence of a particular application. In figure 3 explain the access point in the Bluetooth devices. Most importantly, hover, the physical layer, and the media-access-control (MAC) sub-layer of the data-link layer need to he carefully designed, a job that has fallen on the IEEE 802.11 and 802.5.1 sub groups.

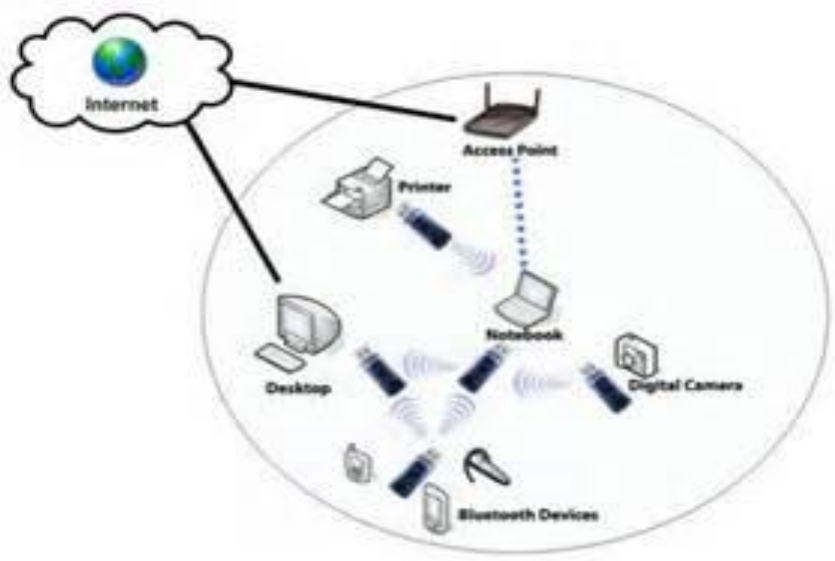

Figure 3:-WPANs

A specific example, wireless devices need to solve a dynamic pro control problem, so that particular device is transmitting at the right pro level: high enough for reliable transmission hut not so high as to interfere with nearby devices for a detailed discussion of this problem).

In addition, the various devices need to cooperate, in order to provide system-wide connectivity. Finally, ideas from game theory have recently introduced to solve the por-control problem on wireless networks.

\section{Description of wireless networks:-}

In this section describe mobile data networks and WLAN's, including existing systems and new systems under development or being defined by standards organizations. The main characteristics of these systems as well as the applications they are intended to serve. Finally, comment on the future directions of technological development in each category of system.

\section{Mobile Data Networks:-}

Packet data services currently available for mobile applications include ARDIS, formed by IBM and Motorola, I404 and the RAM Mobile Data Network, which uses Ericsson MOBITEX data technology. Soon to be introduced into the market is Cellular Digital Packet Data (CDPD), being designed to transport data as a supplementary service overlaid onto existing analog cellular telephone networks. The new digital cellular standards, IS-54 and IS-95, will 
eventually include an may of data services, including both circuit-mode and packet-mode services, for the next generation North American digital cellular systems. In Europe, the European Technical Standards Institute (ETSI) has begun developing a public standard for trunked radio and mobile data systems, designated as TETRA.

\section{Ardis:-}

ARDIS is a two-way radio service developed as a joint venture between IBM and Motorola, and first implemented in 1983. The ARDIS network consists of four network control centers with 32 network controllers distributed through 1250 base station in 400 cities in the U.S. The service is suitable for two-way transfers of data files of size less than 10 kbytes, and much of its use is in support of computer-aided dispatching, such as is used by field service personnel, often while they are on customers' premise. Remote users access the system from lap-top radio terminals, which communicate with the base stations. Each of the ARDIS base stations is tied to one of the 32 radio network controllers. The backbone of the network is implemented with leased telephone lines.

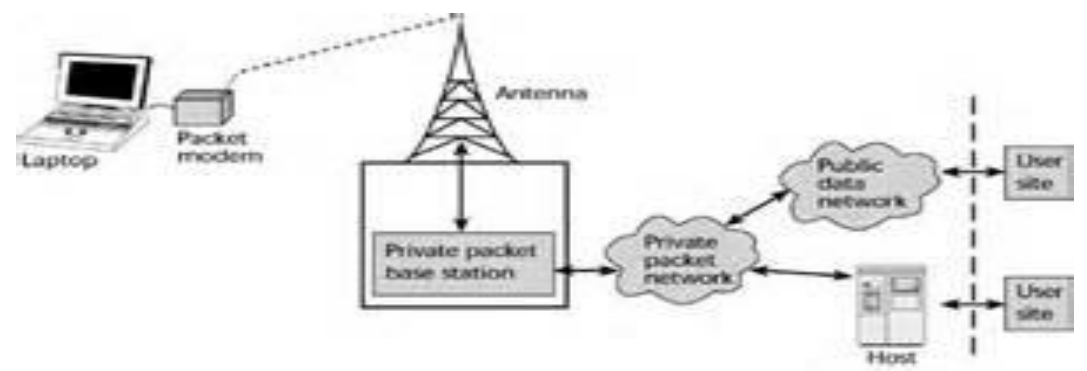

\section{Mobitex:-}

Fig.5:- ARDIS

System is a nationwide, interconnected, trunked radio network developed by Ericsson and Sdish Telecom. The first MOBITEX network into operation in Sden in 1986, and other networks have been implemented in Norway, Finland, Canada, the U. K., and the U.S. In the U.S., MOBITEX service was introduced by RAM Mobile Data in 1991 and now covers almost 100 major metropolitan areas. While the MOBITEX system was designed to carry voice and data services, the U.S. and Canadian networks are used to provide data service only.

MOBITEX is an intelligent network with an open architecture which allows establishing virtual networks. This feature facilitates the mobility and expandability of the network. The system supports a number of standardized network interfaces, including TCP/IP and X.25, and interfaces are added in response to market demands.

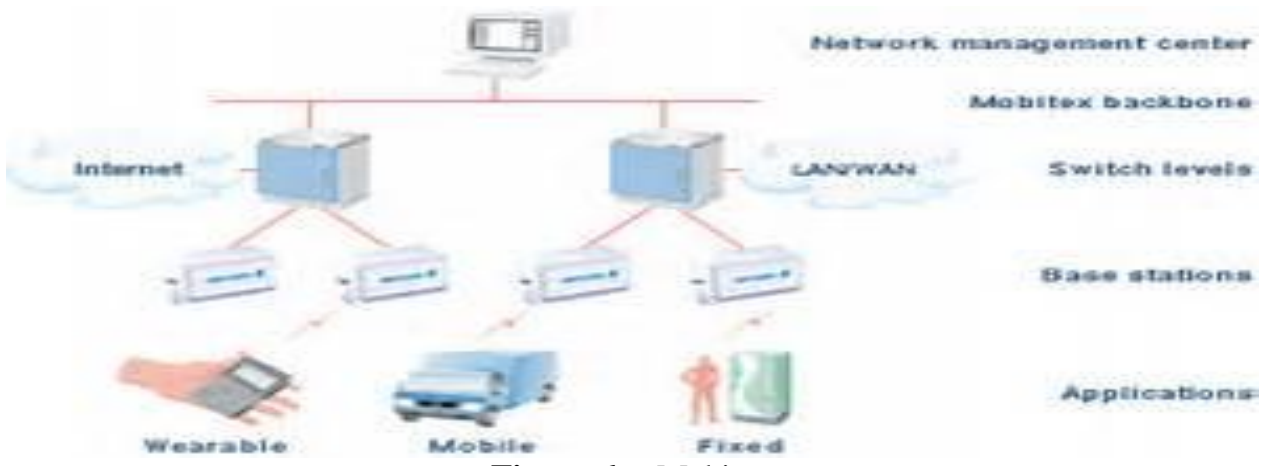

Figure 6:-. Mobitex

The MOBITEX network architecture is hierarchical, At the top of the hierarchy is the Network Control Center (NCC), from which the entire network is managed. The top level of switching is a national switch (MHX1) that routes traffic between service regions. The next level comprises regional switches (MHX2's), and below that is local switches (MOX's), each of which handles traffic within a given service area.

At the lost level in the network, multichannel trunked-radio base stations communicate with the mobile and portable data etc. MOBITEX uses packet-switching techniques, as does ARDIS, to allow multiple users to access the same 
channel at the same time. Message packets are switched at the lost possible network level. If two mobile users in the same service area need to communicate with each other, their messages are relayed through the local base station, and only billing information is sent up to the NCC. The base stations are laid out in a grid pattern using the same system engineering rules as are used for cellular telephone system.

\section{Citations:-}

Wireless Network Evaluation can be briefly explained in [3] and T.S.Rappaport explained about Wireless Communication of Intelligent Networking Architectures. Mobile Cellular Telecommunication System describe in MOBITEX network architecture of the Network Control Center (NCC) in [7].

\section{Conclusions:-}

This paper, explained an overview of wireless LANs and PANS, stressing the two most-common standards, IEEE 802.11 and Bluetooth. While limited in scope, the content attempted to give the reader a quick comparison beten the two technologies, stressing various problem and solutions to wireless networking problems. Current trends in the evolution of wireless data services are either in the direction of high-speed wireless LAN's or low-speed widecoverage mobile data services.

\section{References:-}

1. T.S.Rappaport, "Wireless Communications: Principles and Practice" Upper Saddle River, New Jersey, Prentice Hall, 2002.

2. A. J. Viterbi, "CDMA Principles of Spread Spectrum Communication" Reading, MA, Addison-sley, 1995.

3. V.K.Garg, "Wireless Network Evolution: 2G to 3G, Upper Saddle River, New Jersey, Prentice Hall, 2002.

4. Bluetooth SIG b site: http://www.bluetooth.org.

5. HiperLAN2 Global Forum b site: http://www.hiperlan2.com

6. W. C. Y. Lee, "Mobile Cellular Telecommunications Systems" New York, McGraw Hill, 1989.

7. M. Yacoub, "Foundations of Mobile Radio Engineering" CRC Press, 1993.

8. IEEE, "Wireless Media Access Control (MAC) and Physical Layer (PHY) Specification" P802.11D6.2, July 1998.

9. C.Ganesh,B.Sathiyabama,T.Geetha"Fast Frequent Pattern Mining Using Vertical Data Format for Knowledge Discovery” International Journal of Emerging Research in Management and Technology",Vol 5,issue 5,2016.

10. Dr.N.Muthumani, K.Pavithradevi, "Image Compression Using ASWDR and 3D - SPHIT Algorithms for Satellite Data "International Journal of Science and Engineering Research “, Volume 6, October 2015, PN 289-296. 